\title{
Review of nycteribiid flies (Diptera: Nycteribiidae) of Russia
}

\section{Обзор мух-никтерибиид (Diptera: Nycteribiidae) России}

\author{
G.V. Farafonova ${ }^{1 \dagger}$, N.G. Gornostaev ${ }^{2}$ \\ Г.В. Фарафонова ${ }^{1 \dagger}$, Н.Г. Горностаев ${ }^{2}$
}

\footnotetext{
${ }^{1}$ Department of Entomology, Biological Faculty of Moscow State University, Vorob’evy gory, Moscow, 119899, Russia.

1 Кафедра энтомологии Биологического факультета Московского государственного университета, Воробьевы горы, 119899 , Россия.

${ }^{2}$ N.K.Koltzov Institute of Developmental Biology of Russian Academy of Sciences, Vavilov Street 26, Moscow, 119991, Russia. E-mail: n_gornostaev@mail.ru

${ }^{2}$ Институт биологии развития им. Н.К.Кольцова РАН, ул. Вавилова 26, Москва, 119991, Россия.
}

KEY WORDS: Nycteribiidae, fauna, Russia, new records.

КЛЮЧЕВЫЕ СЛОВА: Nycteribiidae, фауна, Россия, новые указания.

ABSTRACT. A checklist of Nycteribiidae flies from Russia including 18 species of 4 genera is provided. Three species, Basilia nana Theodor, 1954, Nycteribia latreillii Leach, 1817 and N. pedicularia Latreille, 1805 are found in Russia for the first time.

РЕЗЮМЕ. Представлен аннотированный список видов мух семейства Nycteribiidae включающий 18 видов из 4 родов. Три вида, Basilia nana Theodor, 1954, Nycteribia latreillii Leach, 1871 и $N$. pedicularia Latreille, 1805, впервые указаны в фауне России.

\section{Introduction}

The flies Nycteribiidae are known to be parasites of the bats (order Chiroptera) only. The Nycteribiidae includes 3 subfamilies, 12 genera, and about 300 species worldwide [Dick, Patterson, 2006]. Two subfamilies, Archinycteribiinae (1 genus, 3 species), and $\mathrm{Cy}-$ clopodiinae (4 genera, 62 species) are exclusively Eastern Hemispheric in distribution, where they are primarily parasites of bats of the suborder Megachiroptera (mainly associated with the bat family Pteropodidae). The Nycteribiinae (7 genera, 210 species) are cosmopolitan in distribution and they are primarily parasites of bats of the suborder Microchiroptera (mainly associated with the bat families Vespertilionidae and Rhinolophidae). The majority of nycteribiid species are found in tropical and subtropical regions worldwide, however, they are richer in the Eastern Hemisphere (222 species versus 53 species in the Western) [Dick, Patterson, 2006].

Two subfamilies, 34 species and 5 genera are known in Palearctic [Theodor, 1954, 1967; Hůrka, Soós, 1986] and one subfamily, 15 species and 4 genera in Russia [Stackelberg, 1970; Farafonova, 1999].

All these flies are completely wingless. Apparently, the life cycle of nycteribiids is rather uniform. For example, the total life of Basilia hispida Theodor, 1967 is about 136 and 195 days for males and females, respectively, including 9 days in the larval stages, 25 days in puparium, and 97 days (males) or 156 days (females) in the adult stage [Marshall, 1970]. Flies reached sexual maturity 5-6 days after emergence from the puparium. The females deposited prepupae on roost substrate. Prepupal deposition occurred when the bats were in their roosts. From 25 to 46 days later, with host bats present or absent in the roost, wingless adults emerged and walked around searching for a host. The nycteribiids often have a high degree of host specificity and their species diversity depends on the diversity of fauna of the bats.

The fauna of Nycteribiidae of Old World is studied well enough and relatively full species checklists are provided in literature. However, a new checklist of nycteribiids of Russia is absent up to date. The present paper include various materials on the Nycteribiidae from different regions of Russia. All the materials studied are kept in the collection of Department of Entomology, Biological Faculty of Moscow State University.

\section{Checklist of Nycteribiidae of Russia}

Genus Basilia Miranda-Ribeiro, 1903

Basilia nana Theodor, 1954

MATERIAL. $1 \sigma^{7}, 4$ ㅇ, Russia, Krasnodar Terr., near Adler, caves, 1-31.08.1999, from Myotis bechsteini, leg. A. Borisenko; 1

How to cite this article: Farafonova G.V., Gornostaev N.G. 2018. Review of nycteribiid flies (Diptera: Nycteribiidae) of Russia // Russian Entomol. J. Vol.27. No.4. P.435-438. doi: 10.15298/rusentj.27.4.11 
+, Caucasus State Natur Reserve, Laura, 1-31.07.1999, from M. mystacinus, leg. A. Borisenko.

DISTRIBUTION. Russia (new record): North Caucasus. Europe, Caucasus, Israel [Hůrka, Soós, 1986].

HOSTS: Myotis bechsteini Kuhl, 1817, M. dasycneme (Boie, 1825), M. mystacinus Kuhl, 1819, M. nattereri (Kuhl, 1817).

\section{Basilia rybini Hůrka, 1969}

MATERIAL. $6 \sigma^{7} \sigma^{7}, 7$ 7 , Russia, Novosibirsk Prov., Maslyaninsk Distr., caves, 2.05.1981, from M.daubentoni, leg. P. Morozov; $2 \sigma^{\top} \sigma^{\top}, 2$, Far East, Primorsky Terr., cave Seraphimovskaya, 4.02.1983, from M. nattereri, leg. M.P. Tiunov; 1 ऽ, Far East, Primorsky Terr., cave Makrushinskaya, 6.02.1983, from M. nattereri, leg. M.P. Tiunov; $1 \sigma^{\top}$, Far East, Primorsky Terr., cave Velican, 11.03.1983, from M. frater, leg. M.P. Tiunov; $1 \sigma^{7}, 1$, Primorsky Terr., cave Priiskovaya, 13.02.1983. leg. M.P. Tiunov; $1 \Im^{7}$, Primorsky Terr., Khasan, 1-31.03.1983, from M. daubentoni, leg. M.P. Tiunov; 1 ㅇ, Khabarovsk Terr., 18.08.1983, from $M$. daubentoni, leg. M. P. Tiunov; 1 , Khabarovsk Terr., 27.08.1983, from $M$. frater, leg. M.P. Tiunov; 1 $\sigma^{7}, 4$ 9 , Khabarovsk Terr., cave Stary Medved, from M. nattereri; $5 \sigma^{\top} \sigma^{\top}, 4$, 9 , Altai, caves, 25.01-5.02.1989, from M. daubentoni, leg. A. Polkanov.

DISTRIBUTION. Russia: Siberia, Far East [Medvedev et al., 1991; Farafonova, 1999]. Kazakhstan, Turkmenia.

HOSTS: Myotis daubentoni Kuhl, 1819, M. nattereri, M. mystacinus, M. frater G. Allen, 1923.

\section{Basilia truncata Theodor, 1966}

MATERIAL. 1 o', 2 우, Far East, Khasan, Zaozernaya, 131.08.1981, from Miniopterus schreibersi, leg. P. Morozov; $1 \sigma^{7}$ Far East, cave Priiskovaya, 17.12.1983, from Murina leucogaster, leg. M.P. Tiunov; 1 q, Far East, Kiparisovo, 25.07.1985, from M.daubentoni, leg. M.P. Tiunov; $2 \sigma^{7} \sigma^{7}$, Khabarovsk Terr., cave Steregushee Kop'e, 24.10.1983, from M. brandti, leg. M.P. Tiunov; $2 \sigma^{\top} \sigma^{\top}, 2$ 우, Khabarovsk Terr., cave, Proshania, 30.10.1983, from M. brandti, leg. M.P. Tiunov; $2 \sigma^{7} \sigma^{7}, 7$ 7 , Khabarovsk Terr., cave Stary Medved', 30.10. 1983, from M. brandti, leg. M.P. Tiunov; 1 ऽ', Altay, cave, 5.02.1983, from Murina leucogaster, leg. M.P. Tiunov; $2 \sigma^{7} \sigma^{\top}, 4$ 우, Khakasia, caves, 1986, from M.brandti, leg. A. Hritankov; 1 ○, 1 क, Krasnoyarsk Terr., cave Ledopadnaya 22.03.1987, from M. brandti, leg. A. Hritankov; $2 \sigma^{\top} \sigma^{\top}, 2$ 우, from M. daubentoni, Krasnoyarsk Terr., cave Tergalinskaya, 21.12.1989, leg. Kotepov; $1 \sigma^{\top}$, from M. brandti, Krasnoyarsk Terr., cave Podnebesnaya,1-28.02.1984, leg. A. Hritankov.

DISTRIBUTION. Russia: Siberia, Far East [Medvedev et al., 1991; Farafonova, 1999]. Mongolia.

HOSTS: Miniopterus schreibersi Kuhl, 1819, Murina leucogaster Milne-Edwards, 1872, Myotis brandti Eversmann, 1845, M. daubentoni, M. mystacinus.

Basilia truncatiformis Farafonova, 1998

MATERIAL. $2 \sigma^{7} \sigma^{\top}, 3$, 3 , Russia, Krasnoyarsk Terr., Shirinsk Distr., vill. Malaya Siya, cave Archeologicheskaya, 5.10.1989, from M. brandti, leg. A. Hritankov, Denisova; $2 \sigma^{7} \sigma^{7}, 1$, Krasnoyarsk Terr., river Biryusa, caves, 19.09.1989, from M. brandti, leg. A. Hritankov.

DISTRIBUTION. Russia: Siberia [Farafonova, 1998]. HOST: Myotis brandti.

Genus Nycteribia Latreille, 1797

\section{Nycteribia allotopa Speiser, 1901}

MATERIAL. $1 \sigma^{7}, 1$, Russia, from Miniopterus schreibersi, Far East, Primorsky Terr., Khasan, Zaozernaya, 2.08.1988, leg. P. Morozov.

DISTRIBUTION. Russia: Far East [Medvedev et al., 1991; Farafonova, 1999]. Japan, China, Sri Lanka, India. HOST: Miniopterus schreibersi.
Nycteribia formosana (Karaman, 1939)

MATERIAL. $4 \sigma^{\top} \sigma^{\top}, 2$ 우, Russia, no host, Far East, Khasan, 6.07.1966, leg. V.G. Belyaev; $2 \mathrm{O}^{\top} \mathrm{O}^{\top}, 2$ 우 from Miniopterus schreibersi, Far East, Khasan, Zaozernaya, 2.08.1981, leg. P. Morozov; $3 \bigcirc^{7} \sigma^{7}, 3$ 우 from Myotis capaccini, Far East, cave Seraphimovskaya, 5.06.1983, leg. M.P. Tiunov; $1 \mathrm{O}^{7}, 1$ ㅇ from M. daubentoni, Far East, cave Velican, 11.03.1983, leg. M. Tiunov; 2 우우 from Plecotus auritus, Far East, cave Bogataya Fanza, 7.03.1983, leg. M. P. Tiunov; 1 , from M. capaccini, Far East, cave Velican, 11.03.1983, leg. M.P. Tiunov; 2 qO, from M. daubentoni, Far East, cave Letuchaya mish, 14.06 .1985 , leg. M.P. Tiunov; $5 \sigma^{7} \sigma^{7}, 4$ 우, from M. capaccini, Far East, Khasan, Zaozernaya, 18.07.1985, leg. M.P. Tiunov; $1 \sigma^{7}$, from M. cappacini, Sachalinskaya ar., Kunashir, 2.08.1985, leg. M.P. Tiunov.

DISTRIBUTION. Russia: Far East [Medvedev et al., 1991; Farafonova, 1999]. China, Taiwan (Formosa).

HOSTS: Miniopterus schreibersi, Myotis capaccini Bonaparte, 1837, M. daubentoni, M. ricketti Thomas, 1894, Plecotus auritus (Linnaeus, 1758), Rhinolophus ferrumequinum Schreber, 1775.

Nycteribia kolenati Theodor et Moscona, 1954

MATERIAL. 16 O $^{\top} \sigma^{7}, 20$ 우, Russia, from Vespertilio sp., Simbirsk Prov. 07.1896, leg. Pleske, det. Stackelberg; 9 O$^{7} \bigcirc^{7}, 14$ 우, from Myotis daubentoni, Penza Prov., Novo-Lomovsky rest., vill.Virta, 1-30.09.1983, leg.V.Yu. Il'in; $1 \sigma^{7}$, from M. brandti, 13.12.1977, leg. V.Yu. Il'in; 5 우, from M. daubentoni, Penza, Norovschatsk Distr., vill. Skan, 1-31.08.1983, leg. V.Yu. Il'in; 6 $\mathrm{O}^{\top} \mathrm{O}^{\top}, 6$ 우, from M.daubentoni, Krasnodar Ter., Adler Distr., 131.08.1999, leg. A. Borisenko; Pskov Prov., $2 \mathrm{O}^{7} \mathrm{O}^{7}, 3$ 우, from Myotis dasycneme, 1-30.04.1984, leg. Masing.

DISTRIBUTION. European part of Russia and Ural [Markova, 1938; Orlova, 2011]. Caucasus, Kazakhstan.

HOSTS: Myotis daubentoni, M. nattereri, M. brandti, M. dasycneme, Vespertilio murinus Linnaeus, 1758, Eptesicus serotinus (Schreber, 1774).

Nycteribia latreillii (Leach, 1817)

MATERIAL. $1 \sigma^{7}$, Russia, North Osetiya, Alagir, 13.06.1982, from M. blythi, leg. S. Alekseev; $6 \mathrm{O}^{7} \sigma^{7}, 6$ + 9 , from M. daubentoni, Krasnodar Terr., Adler Distr., caves, 1-31.08.1999, leg. A. Borisenko.

DISTRIBUTION. Russia (new record): North Caucasus. Europe, North Africa, West Asia.

HOSTS: Myotis daubentoni, M. blythi Tomes, 1857, M. myotis Borkhausen, 1797, Rhinolophus ferrumequinum.

\section{Nycteribia pedicularia Latreille, 1805}

Material. $1 \mathrm{O}^{7}, 2$ 웅, Russia, Altai, cave, 1-31.08.1989, from M. daubentoni, leg. A. Polkanov; $1 \sigma^{7}, 4$ 우, Adygeya, 131.07.1985, from Miniopterus schreibersi, leg. S. Alekseev.

DISTRIBUTION. Russia (new record): North Caucasus, Altai. Europe, North Africa, West Asia.

HOSTS: Miniopterus schreibersi, Myotis daubentoni, M. myotis, M. capaccini, Rhinolophus ferrumequinum.

Nycteribia pygmaea (Kishida, 1932)

MATERIAL. $5 \bigcirc^{7} \sigma^{7}, 4$ 오, Russia, Magadan Prov., Ol'sk Distr., riv. Shelomdga, 1-31.08.1981, from M. daubentoni, leg. M.E. Dokushaev; $2 \sigma^{7} \sigma^{7}, 1$ ㅇ, 14.08.1982, from M. daubentoni, leg. M. Dokushaev; $750^{7} \sigma^{7}, 108$ 우, no host, Far East, Khasan, 131.07.1966, leg. Belyaev; $1 \sigma^{7}$, Far East, Khasan, Zaozernaya, 2.08.1981, from Miniopterus schreibersi, leg. P. Morozov.

DISTRIBUTION. Russia: Far East [Medvedev et al., 1991; Farafonova, 1999]. Japan, Korea.

HOSTS: Miniopterus schreibersi, Myotis daubentoni, Rhinolophus ferrumequinum, Pipistrellus savii (Bonaparte, 1837). 
Nycteribia quasiocellata Theodor, 1966

MATERIAL. $150^{7} \sigma^{7}, 18$ 우, Russia, Novosibirsk Prov., Maslyaninsky Distr., caves, 2.05.1981, from M. daubentoni and M. mystacinus, leg. P. Morozov; $1 \sigma^{\top}, 3$ +क , from M. daubentoni, Altai, cave, 1-31.03.1986, leg. A. Polkanov; 1 9 , Chita Prov., cave Kira, 1-31.07.1988, from Eptesicus nilssoni, leg. A. Hritankov; 1 O$^{7}, 5$ 우, Krasnoyarsk Terr., Schugur, 1988, from M. mystacinus, leg. A Hritankov; 2 O $^{\top}$, 2 우, Krasnoyarsk Terr., cave Ledopadnaya, 131.03.1987, from M. daubentoni, leg. A. Hritankov; $2 O^{7} O^{7}, 2$ 우, Krasnoyarsk Terr., Malaya Siya, cave Archeologycheskaya, 5.10 1989, from M. daubentoni, leg. A. Hritankov; $2 \bigcirc^{7} \bigcirc^{7}$, Krasnoyarsk Terr., cave Torgalinskaya, 21.12.1989, from $M$. daubentoni, leg. Kotepov; $20^{7} \sigma^{7}, 3$ 우, Khabarovsk Terr., Blagoveschensk, mansarda of house, 12.06.1984, from M. daubentoni, leg. M.P. Tiunov; $9 \mathrm{O}^{7} \mathrm{O}^{7}, 7$ 우, Ayano-Maysky Distr., caves, 1-31.08.1984, from M.daubentoni, leg. M. Tiunov.

DISTRIBUTION. Russia: Siberia, Far East [Medvedev et al., 1991; Farafonova, 1999; Orlova et al., 2014]. Mongolia, Kazakhstan, China.

HOSTS: Myotis daubentoni, M. mystacinus, Eptesicus nilssoni Keyserling et Blasius, 1839.

Nycteribia schmidlii Schiner, 1853

MATERIAL. $10^{\top}, 2$ 우, Russia, Krasnodar Terr., near Sochi, 1-31.07.1978, from Miniopterus scheibersi, leg. P. Sagdieva; $10^{7}$ 3 오, Simbirsk Prov., 1-31.08.1896, no host, det. A.A. Stackelberg, leg. Pleske; $1 \sigma^{7}, 4$ 우, Krasnodar Terr., near Adler, cave, 19.08.1979, from Pipistrellus sp., leg. M. Perov.

DISTRIBUTION. Russia: North Caucasus, Central Volga, Crimea [Stackelberg, 1970]. Caucasus. Austria, Turkey, Lebanon, Israel, Iran, Afghanistan, Algeria.

HOSTS: Miniopterus schreibersi, Rhinolophus ferruequinum, Rh. euryale Blasius, 1853, Plecotus auritus, Pipistrellus sp.

Nycteribia vexata Westwood, 1835

MATERIAL. $20^{7} \sigma^{7}, 3$ 우, Russia, Krasnodar Terr., near Adler, cave, 19.08.1979, from Myotis sp., leg. M. Perov.

DISTRIBUTION. Russia: North Caucasus, Crimea [Volkova, 1957]. Caucasus, West Europe, North Africa, West Asia. tus.

HOSTS: Myotis blythi. Rhinolophus spp., Plecotus auri-

Genus Penicillidia Kolenati, 1863

\section{Penicillidia conspicua Speiser, 1901}

MATERIAL. $1 \sigma^{\top}$, Russia, from Vesperugo sp., Simbirskaya Prov., 12.08.1896, leg. Pleske, det. A. Stackelberg.

DISTRIBUTION. Russia: Central Volga, Tatarstan, Zabaikal'e [Volkova, 1957; Jovty et al., 1962; Stackelberg, 1970]. Europe, North Africa.

HOSTS: Miniopterus schreibersi, Myotis spp., Rhinolophus sp., Vesperugo sp.

Penicillidia dufouri Westwood, 1835

MATERIAL. 1 9, Russia, Simbirskaya Prov.,12.08.1896, no host, leg. Pleske, det. Stackelberg; $1 \mathrm{O}^{\top}$, Novosibirsk Prov., caves, 2.05.1981, from $M$. daubentoni, leg. P. Morozov; $1 \sigma^{7}$, Penza Prov., Novo-Lomovsky Distr., vill. Virga, 11.07.1983, from $M$. daubentoni, leg. V.Yu. Il'in; 1 O', 1 +, Tver' Prov., cave, 1985, from $M$. daubentoni, leg. K.K. Panyutin; $1 \sigma^{7}, 1$, Altai, 128.02.1989, from M. dasycneme, leg. A. Polkanov.

DISTRIBUTION. Russia: European part, West Siberia, Far East [Stackelberg, 1970; Medvedev et al., 1991; Farafonova, 1999]. Europe, Japan, China, Middle Asia.

HOSTS: Myotis daubentoni, M. dasycneme, Rhinolophus spp., Miniopterus schreibersi.
Penicillidia jenynsii (Westwood, 1835)

MATERIAL. $2 \sigma^{7} \sigma^{7}$, Russia, Far East, Khasan, 7.07.1966, from Miniopterus schreibersi, leg. V.G. Belyaev, $20^{7} \sigma^{7}, 4$, 99 , Far East, Khasan, Zaozernaya, 26.07.1981, from M. schreibersi, leg. P, Morozov; 1 O$^{7}, 1$ ㅇ, 2.08. 1981, from M. schreibersi, leg. P. Morozov.

DISTRIBUTION. Russia: Far East [Medvedev et al., 1991; Farafonova, 1999]. Japan, China, Taiwan.

HOST: Miniopterus schreibersi.

\section{Penicillidia monoceros Speiser, 1900}

MATERIAL. $2 \sigma^{7} \sigma^{7}, 1$ \% , Russia, Novosibirsk Prov., cave, 2.05.1981, from $M$. daubentoni, leg. P. Morozov; 4 우, Altai, 25.01-5.02.1989, from M. daubentoni, leg. A. Polkanov; $20^{7} \sigma^{7}, 1$ +, Far East, Primorsky Terr., cave Sinegorskaya, 6.11.1983, from M. nattereri, leg. M. Tiunov; $1 \sigma^{7}, 19$, Far East, Primorsky Terr., cave Seraphimovskaya, 4.02.1983, from M. nattereri, leg. M.P. Tiunov; 1 , Khabarovsk Terr., Ayano-Maisky Distr., Abogy-Dyus, 13.08.1984, from M. daubentoni, leg. M.P. Tiunov; $2 \sigma^{7} \sigma^{1}, 4$ 오, Tver' Prov., quarry, 28.03.1985, from M. dasycneme, leg. P. Morozov.

DISTRIBUTION. Russia: European part, Siberia, Far East [Markova, 1938; Stackelberg, 1970; Medvedev et al., 1991; Farafonova, 1999]. Mongolia.

HOSTS: Myotis daubentoni, M. nattereri, M. dasycneme, Plecotus auritus, Eptesicus nilssoni.

\section{Genus Phthiridium Hermann, 1804}

Phthiridium biarticulatum (Hermann, 1804)

MATERIAL. $1 \sigma^{7}$, Russia, Krasnodar Terr., caves, 1-31.08. 1976, from Rhinolophus sp., leg. M. Perov.

DISTRIBUTION. Russia: North Caucasus [Stackelberg, 1970]. Europe, North Africa, West Asia.

HOSTS: Rhinolophus ferrumequinum, Miniopterus schreibersi, Pipistrellus pipistrellus (Schreber, 1774).

\section{Discussion}

Thus, a total of 18 species (genera Basilia, Nycteribia, Penicillidia and Phthiridium) of subfamily Nycteribiinae are found in Russia, it is more than half of 34 species of this subfamily known from Palearctic. Three species, Basilia nana, Nycteribia latreillii and Nycteribia pedicularia, are found in Russia for the first time. Thus, 9 nycteribiid species recorded in the Far East (Basilia rybini, B.truncata, Nycteribia allotopa, N. formosana, N. pygmaea, $N$. quasiocellata, Penicillidia dufouri, $P$. jenynsii, P.monoceros), 8 species in Siberia (Basilia rybini, B. truncata, B.truncatiformis, Nycteribia quasiocellata, $N$. pedicularia, Penicillidia conspicua, $P$. dufouri, $P$. monoceros), 7 species in the North Caucasus (Basilia nana, Nycteribia kolenati, $N$. latreillii, $N$. pedicularia, $N$. schmidlii, $N$. vexata, Phthiridium biarticulatum) and 5 species in the center of European Russia (Nycteribia kolenati, N. schmidlii, Penicillidia conspicua, P. dufouri, P. monoceros).

\section{References}

Dick C.W., Patterson B.D. 2006. 11. Bat flies: Obligate ectoparasites of bats // Morand S., Krasnov B.R., Poulin R. (eds.). 
Micromammals and Macroparasites: From Evolutionary Ecology to Management. Tokyo. Springer. P.179-194.

Farafonova G.V. 1998. [New species of Nycteribiid flies of the genus Basilia Miranda Ribeiro, 1903 (Diptera, Nycteribiidae)] // Entomol. Obozr. Vol.77. No.1. P.224-228 [in Russian].

Farafonova G.V. 1999. [Family Nycteribiidae] // Lehr P.A. (ed.). Opredelite' nasekomykh Dal'nego Vostoka Rossii. Vol.6. Dvukrylye i blokhi. Pt.1. Vladivostok: Dal'nauka. P.581-588 [in Russian].

Hůrka K., Soós A. 1986. Nycteribiidae // Soós Á., Papp L. (eds.). Catalogue of Palaearctic Diptera. Vol.11. Amsterdam: Elsevie Science Publishers, Budapest: Akadémiai Kiadó. P.26-234

Jovty I.F., Zarubina V.N., Prokop'ev V.N., Schvedko L.P. 1962. [Ectoparasites of the bats in the South-Eastern Transbaikalia and adjacent areas in Mongolia] // Izvestiya Irkutskogo nauchno-issledovatel'skogo protivochumnogo instituta. Sibiri i Dal'nego Vostoka Vol.24. No.9. P.338-343 [in Russian].

Markova L.I. 1938. [Influence of winter hibemation on parasitofauna of bats] // Zool. Zhurn. Vol.7. P.33-145 [in Russian].

Marshall A.G. 1970. The life cycle of Basilia hispida Theodor 1957 (Diptera: Nycteribiidae) in Malaysia // Parasitology. Vol.61. P.1-18.

Medvedev S.G., Stanukovith M.K., Tiunov M.P., Farafonova G.V. 1991. [Ectoparasites of bats from the Far East] // Parasitologiya. Vol.25. No.1. P.27-37 [in Russian].
Orlova M.V. 2011. Ectoparasites of the pond bat (Myotis dasycneme (Boie, 1825), Chiroptera) in the Urals // Fundamental'nye problemy entomologii v XXI veke.Materialy mezhdunarodnoy nauchnoy konferentsii. Saint-Petersburg: Saint-Petersburg State University Publishing House. P.123.

Orlova M.V., Orlov O.L., Zhigalin A.V. 2014. New records of ectoparasites of the eastern water bat Myotis petax Hollister, 1912 (Vespertilionidae, Chiroptera) and the revision of the material previously collected from Myotis daubentonii s. lato in the Eastern Palaearctic // Entomol. Rev. Vol.94. No.9. P.1306-1312.

Stackelberg A.A. 1970. [Fam. Nycteribiidae] // Opredelitel' nasekomykh Evropeiskoi chasti SSSR. Diptera and Siphonaptera. Vol.5. Pt.2. P.603-607 [in Russian].

Theodor O. 1954. Familie 66a: Nycteribiidae // Lindner E. (Hrsg.). Die Fliegen der palaearktischen Region. Stuttgart. Bd.8. S.144.

Theodor O. 1967. An illustrated catalogue of the Rothschild collection of Nycteribiidae (Diptera) in the British Museum (Natural History). London. 506 p.

Volkova L.I. 1957. [Bloodsucking flies of Tatar and Chuvash Republics] // Uchenye zapiski Kazanskogo gosudarstvennogo universiteta. Vol.117. No.2. P.241-245 [in Russian]. 